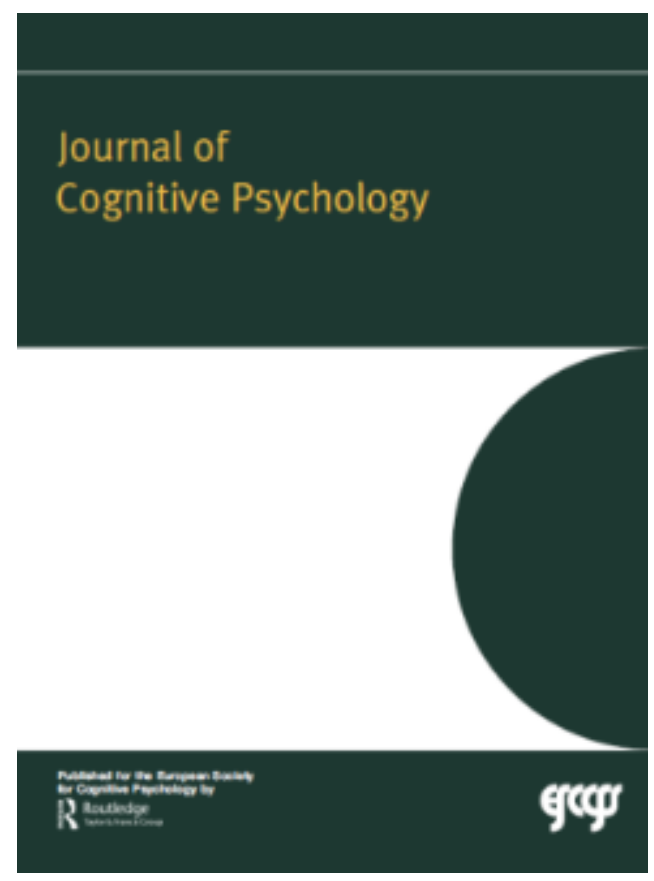

\title{
Right-hemisphere coherence to speech at pre-reading stages predicts reading performance one year later
}

\begin{tabular}{|r|l|}
\hline Journal: & Journal of Cognitive Psychology \\
\hline Manuscript ID & JCP-FA 21-24.R1 \\
\hline Manuscript Type: & Full Article \\
\hline Author: & n/a \\
\hline & $\begin{array}{l}\text { Complete List of Authors: } \\
\text { Lourinaro, Nicola; BCBL, } \\
\text { Bovelpment Grounon, Mathieu; Université Libre de Bruxelles, Laboratoire de } \\
\text { Cartographie Fonctionnelle du Cerveau; BCBL } \\
\text { Lallier, Marie; BCBL }\end{array}$ \\
\hline Keywords: & $\begin{array}{l}\text { Speech-brain coherence, Reading acquisition, Cognitive development, } \\
\text { EEG, Brain oscillations }\end{array}$ \\
\hline &
\end{tabular}

\section{SCHOLARONE \\ Manuscripts}




\section{Right-hemisphere coherence to speech at pre-reading stages}

2 predicts reading performance one year later

3 Authors: Paula Ríos-López ${ }^{123^{*}}$, Nicola Molinaro ${ }^{14}$, Mathieu Bourguignon ${ }^{15}$ and

4 Marie Lallier $^{1}$

5

6 1BCBL, Basque Center on Cognition, Brain and Language, Donostia/San Sebastian,

7 Spain

$8 \quad$ 2Leibniz Institute for Neurobiology, Magdeburg, Germany

$9 \quad{ }^{3}$ Centre for Behavioral and Brain Sciences, Magdeburg, Germany

$10{ }^{4}$ Ikerbasque, Basque Foundation for Science, Bilbao, Spain

11 5Laboratoire de Cartographie Fonctionnelle du Cerveau, Université Libre de Bruxelles,

12 Bruxelles, Belgium

13

14 * Corresponding author:

15 Paula Ríos-López

16 Leibniz-Institut für Neurobiologie (LIN)

17 Brenneckestraße 6

18 39118, Magdeburg

19 Germany

20 prioslop@lin-magdeburg.de 
1 Abstract

The hypothesis that neural entrainment to the low-frequency modulations of speech contributes significantly to reading acquisition receives increasing support in the literature. Still, no previous study has actually attempted to establish a longitudinal link between them. The present study tested Basquespeaking children twice: once before reading was formally instructed $\left(t_{1} ; 5-6\right.$ years old) and once after they had received a full school year of reading instruction ( $t_{2} ; 6-7$ years old). At $t_{1}$, speech-brain coherence was recorded via EEG. At $t_{2}$, in addition to the coherence measure, reading performance was assessed. Our results show that children with larger pre-reading delta-band $(<1$ $\mathrm{Hz}$ ) speech-brain coherence at right sites of the scalp performed better in the reading tasks one year later. Moreover, the increase in coherence from prereading into reading stages tended to correlate negatively with reading outcome. The latter result, though statistically weak, suggests that reading instruction might lead to the recruitment of less delta tracking resources. Overall, our results provide preliminary support for a relevant contribution of right-hemisphere speech-brain coherence to successful reading development and point towards pre-reading neural coherence indexes as useful tools for the early detection of developmental reading disorders.

\section{Keywords}

Reading acquisition; Speech-brain coherence; EEG; Cognitive development; Brain oscillations 


\section{Introduction}

Along the last two decades, neural oscillations have been ascribed an increasing number of functions for language processing, from the sheer segmentation of syllables in the speech stream to higher linguistic functions such as syntactic computations (Meyer, 2017). Thus, hypotheses such as the asymmetric sampling in time (AST) (Boemio, Fromm, Braun, \& Poeppel, 2005; Poeppel, 2003; Poeppel, Idsardi, \& Van Wassenhove, 2008) have proposed that the functional difference of neural oscillations at distinct frequencies is coded in cerebral asymmetries, such that the right hemisphere synchronises preferably to amplitude modulations at the syllable rate $(\sim 4 \mathrm{~Hz}$; theta band), whereas phonemic modulations $(\sim 30 \mathrm{~Hz})$ would be preferentially processed in the left hemisphere or bilaterally. On top of that, although the phrasal/stress rate ( $<4 \mathrm{~Hz}$; delta band) was underrepresented in the original AST proposal, its relevance for speech processing has been supported by multiple studies evaluating neural oscillations in response to speech (Bourguignon et al., 2013; Gross et al., 2013; Keitel, Gross, \& Kayser, 2018; Molinaro \& Lizarazu, 2018).

Importantly, evidence for the role that neural oscillations might play for speech perception and language comprehension comes mostly from adult populations, whereas the question of its development remains largely underinvestigated. Nonetheless, if oscillations are to have a real functional meaning for speech perception, speech-abled young children should already show oscillatory neural sensitivity to speech. This contemplation was corroborated by a recent 3year longitudinal EEG study reporting that children already showed significant speech-brain coherence at four years of age, and that coherence increased significantly with age in bilateral temporal sites of the scalp (Ríos-López, Molinaro, Bourguignon, \& Lallier, 2020). Also, speech-brain coherence occurred only in the delta band frequency $(<1 \mathrm{~Hz})$, a result that is in line with proposals claiming that, at early stages of development, speech perception relies more on slow speech information as opposed to phonemic contrasts (Anthony \& Francis, 2005; Ziegler \& Goswami, 2005). 
Interestingly, in the study by Ríos-López et al. (2020), speech-brain coherence in the right hemisphere (but not in the left) was related to an indirect measure of intelligibility, such that the larger the coherence value, the greater the accuracy in answering to a series of yes/no comprehension questions related to the speech stimulus. Unfortunately, due to the children's young age, this was the only intelligibility measure collected, and the question hence remains open as to if the coherence phenomenon is related to other linguistic processes such as reading acquisition.

Indeed, when looking for putative linguistic correlates of delta-band brain oscillations, reading comes up as a putative candidate, since right-hemisphere entrainment to the slow rhythms of speech has been assigned a causal role for its successful acquisition (Goswami, 2011, 2018; Lallier et al., 2018; Lallier, Molinaro, Lizarazu, Bourguignon, \& Carreiras, 2017). Succinctly, the oscillatory temporal sampling framework (Goswami, 2011) proposes that abnormal righthemisphere processing of the speech amplitude fluctuations at the syllabic and the prosodic rates could lead to abnormal segmentation of the speech signal, which could in turn result in atypical phonemic processing in the left hemisphere and to subsequent reading disorders (for a link between low-frequency neural entrainment and phoneme recognition in natural speech, see Kösem et al., 2018)

This theory has received support from several neurophysiological studies reporting that dyslexic children and adults show abnormal right-hemisphere delta or theta entrainment to the amplitude envelope of speech (Abrams, Nicol, Zecker, \& Kraus, 2009; Molinaro, Lizarazu, Lallier, Bourguignon, \& Carreiras, 2016; Power, Colling, Mead, Barnes, \& Goswami, 2016). There is also evidence that dyslexic children show atypical patterns of right-hemisphere delta- and theta-band entrainment to the phonetic and spectral features of speech, and that this measure correlates with phonological abilities (i.e., phonological awareness, RAN, etc.; Di Liberto et. al., 2018). Nevertheless, none of this evidence is sufficient to prove a causal link between entrainment to the lowfrequency modulations of speech and reading acquisition; all these studies compared participants who could already read (more or less successfully) and, 
evidently, when reading and neural synchronisation to speech are tested concomitantly, bidirectional influences cannot be ruled out.

To our knowledge, the only attempt to establish a longitudinal relation between neural synchronisation and reading ability comes from a study following up typically developing children and children at family risk of dyslexia -some of whom later developed the disorder- from pre-reading into reading stages and measuring their auditory steady state responses to amplitude modulated signals at 4, 20 and $80 \mathrm{~Hz}$ (De Vos, Vanvooren, Vanderauwera, Ghesquière, \& Wouters, 2017). This study reported interesting developmental results, such that the responses of the children who later developed dyslexia to the $20 \mathrm{~Hz}$ (approx. phonemic rate) increased more from the pre-reading into the reading stage as compared to the typically developing children, maybe as a compensatory mechanism during reading instruction. In any case, this study used perfectly periodic artificial rhythms, a stimulus that has received some criticism. For instance, entrainment to perfectly periodic artificial rhythms cannot be considered as a proxy of speech sensitivity, since speech rhythms are characterised by the absence of perfect periodicity (Alexandrou, Saarinen, Kujala, \& Salmelin, 2018). To our knowledge, no study has directly measured pre-reading entrainment to the slow rhythms of natural speech and tested its potential to predict future reading abilities.

\section{The present study}

Here, we examined if delta $(<1 \mathrm{~Hz})$ speech-brain entrainment was related to reading acquisition in typically developing children. We tested children before $\left(t_{1} ; 5-6\right.$ years of age) and after $\left(t_{2} ; 6-7\right.$ years of age $)$ reading was introduced in the school curriculum. Speech-brain coherence derived from EEG recordings was assessed at both testing times, while reading achievement was assessed only at $t_{2}$.

Based on previous theories and on studies in older children and adults (Abrams et al., 2009; Goswami, 2011; Hämäläinen, Rupp, Soltész, Szücs, \& Goswami, 2012; Lizarazu et al., 2015; Molinaro et al., 2016; Power et al., 2016), 
1 we hypothesised that low delta $(<1 \mathrm{~Hz})$ speech-brain entrainment in the right

2 hemisphere at pre-reading stages $\left(t_{1}\right)$ would predict reading variability after

3 reading instruction had been formally introduced $\left(t_{2}\right)$. We also explored the putative relation between reading and the difference in coherence from the prereading $\left(t_{1}\right)$ to the reading $\left(t_{2}\right)$ phase, although our predictions here were not

6 definite, due to the absence of previous evidence in the field.

\section{Materials and methods}

\subsection{Participants}

The children who participated in this study were recruited from two public schools in Donostia-San Sebastián (Spain) and were taking part in a larger three-year longitudinal project. Children were always tested during the summer holidays, that is, in between school years (from mid-June to mid-September). For the present study, we used the data collected at the middle (testing time 1; $t_{1}$ ) and final phase (testing time $2 ; t_{2}$ ) of the larger longitudinal project. With regard to school year, the data at $t_{1}$ were collected after children had finished the last year of pre-school, and the data at $t_{2}$, after children had completed Grade 1. Although all the children were Basque-Spanish bilinguals, they received formal reading instruction in Basque.

At $t_{1}$, none of the children could read (not even at the level of the syllable), according to a brief interview with the parents and the child and with a short test in which words were shown and the child was asked if they knew how to read them.

Children came from a similar socioeconomic background (medium or medium-high) based on their parents' or tutors' yearly net income. They were always tested under signed parental authorization, and the experiment was approved by the BCBL Ethics Review Board and complied with the guidelines of the Helsinki Declaration.

No cognitive anomaly was evident in any of the children, and parents reported children's normal hearing and normal or corrected-to-normal vision. None of the parents reported a family history of developmental language disorder or of any other cognitive pathology. 
1 From the original sample of 42 children who participated in the larger

2 longitudinal study, 31 completed the reading tasks at $t_{2}$ (age $M=6.98, S D$

$3=.32 ; 16$ males). Of them, twenty-nine also completed the EEG task at $t_{1}$ (age $M$

$4=5.87 ; S D=.31 ; 16$ males), and all at $t_{2}$. Finally, 25 children completed the

5 EEG task both at $t_{1}$ and $t_{2}$ and the reading tasks at $t_{2}$. Amongst the whole group,

6 four of the children were left-handers.

\subsection{Stimuli and procedure}

At $\mathrm{t}_{1}$, children came once to the lab to complete the EEG (approx. $1.5 \mathrm{~h}$ ) and the behavioural IQ task (approx. 5 minutes). At $\mathrm{t}_{2}$, the reading task (approx. $15 \mathrm{~min}$ ) and the EEG task (approx. $1.5 \mathrm{~h}$ ) were completed in different sessions to avoid children's fatigue. In both occasions, children performed other linguistic tasks as part of the larger longitudinal project.

\subsubsection{Behavioural battery}

\subsubsection{Neuropsychological screening: IQ assessment}

We obtained a measure of non-verbal IQ by means of the Matrix reasoning subtest from the WPPSI-III (Wechsler, 2002) at each testing time, but only the results at $t_{1}$ were included in subsequent analyses. Children were presented with a matrix of three drawings and a blank square and were asked to complete the series with the correct element out of four to five alternatives. The test consisted of a total of 29 items, and the initial item was adapted upon the child's age (in months). This task lasted approximately ten minutes. Direct scores were converted into scalar (standardised) scores to correct for age differences in months.

\subsubsection{Reading assessment}

At $t_{2}$, two tasks were used to measure two different aspects of reading: Word and pseudoword reading and Text reading.

\subsection{Word and pseudoword reading}


Stimuli were presented in the centre of a CRT computer screen in capital letters in Arial font size 44. Children were instructed to read as fast as possible while minimizing mistakes. The experimenter pressed a button as soon as the child produced a response, which prompted the display of the next item in the computer screen. The time elapsed between button presses was recorded in order to obtain a measure of reading time per item. Stimuli consisted of 20 highfrequency Basque words and 20 pseudowords. Pseudowords were created changing one phoneme of an existing high-frequency word (e.g., LIBUKU from LIBURU [book $\left.\left.{ }^{*}\right]\right)$. Within each category, stimuli varied in length, such that 10 items were bi-syllabic, and the 10 remaining items were tri-syllabic. Five of the items in each condition were formed by syllables with a consonant-vowel structure (e.g., KATU [cat]), while the remaining five contained one syllable with a consonant cluster (e.g., TEKLATU [keyboard]). Total reading time and errors were recorded, and an item per-minute efficiency measure was calculated as follows:

$$
\text { Item reading efficiency }=\frac{\text { Total number of correctly read items }}{\text { Total time }[\mathrm{min}]}
$$

\subsubsection{Text reading}

Participants were asked to read a short passage from the novel Printze Txikia (The Little Prince, written by Saint Exupéry) translated into Basque, a text that has been previously used for other reading studies (Lallier, Valdois, LassusSangosse, Prado, \& Kandel, 2014; Ríos-López, Molnar, Lizarazu, \& Lallier, 2017). The text consisted of 85 words and seven lines. Participants were instructed to read fast, but with as few mistakes as possible. Time (in seconds) and number of errors were recorded. The maximum reading time allowed was 300 seconds. For children who exhausted this time, the number of read words was also recorded. With the time and the errors measures, we calculated the following word perminute efficiency index:

$$
\text { Text reading efficiency }=\frac{\text { Total number of correctly read items }}{\text { Total time }[\mathrm{min}]}
$$




\subsubsection{Vocabulary size at $\mathrm{t} 1$ : Picture naming}

The expressive vocabulary subtest of the Basque English and Spanish Test (BEST; de Bruin, Carreiras, \& Duñabeitia, 2017) was used to control for vocabulary size at $\mathrm{t} 1$. The children saw a total of 39 images, one at a time, in a computer screen. Pictures were coloured, child-friendly images depicting common substantives varying in difficulty. The children's task was to name the pictures. The next picture was only presented after the child emitted a response, and accuracy scores were recorded (maximum score 39). In the context of the larger longitudinal project, the task was completed in Basque and Spanish. However, for the purpose of the present work, only the score in Basque was analysed.

\subsubsection{EEG data recording and task}

We recorded the EEG signals in an electrically-shielded room, where children sat comfortably in front of a computer screen. Children were instructed to stay silent and to refrain from moving as much as they could. EEG signals were recorded using a Brain Products $\mathrm{GmbH}$ 32-electrode actiCAP. Reference electrodes were placed on both mastoids (A1 and A2), and the array of scalp electrodes was reduced to 19 electrodes (FP1, FP2, F7, F3, Fz, F8, F4, C3, Cz, C4, T7, T8, P7, P3, Pz, P8, P4, O1 and O2) distributed over the scalp based on 10-10 International System. This was done in order to reduce preparation time and avoid children's fatigue. In addition, we recorded electrodes FCz and left Heog, right Heog and left Veog. Signals were band-pass filtered between 0.1 $\mathrm{Hz}$ and $150 \mathrm{~Hz}$ and sampled at $500 \mathrm{~Hz}$. Monopolar differential recording was referenced online to electrode FCz.

Children were instructed to listen attentively to natural speech in Basque while staring at an image that remained static on the centre of the screen. In order to avoid familiarity with the story, the participants listened to a different (unknown) story at $t_{1}$ and at $t_{2}$. The different stories were read by the same female speaker and similar loudness was further ensured by normalizing the stimuli to identical RMS level. The stories were uttered at approximately the same syllabic rate (t1: 3.2 syllables/seconds; t2: 3.6 syllables/seconds). The 
1 speaker was instructed to speak at a normal speed and intonation (i.e., avoiding

2 infant directed speech), and the stories were recorded with a sampling

3 frequency of $44.1 \mathrm{kHz}$.

The stories lasted six minutes and were partitioned in one-minute segments. To ensure that children were paying attention, they were asked a yes/no comprehension question after each segment (e.g., Did the eagle come back home?). We also used these recesses to allow the children to rest and (partially) move before resuming the experiment.

The software Psychopy (Peirce, 2009) was used to deliver the speech through loudspeakers at an $80 \mathrm{~dB}$ SPL. The loudspeakers were placed the right and left of the computer screen at an approximate distance of $60 \mathrm{~cm}$ from the child's head.

\subsection{Data analysis}

\subsubsection{Reading tasks}

To ensure that the pseudoword and word reading subtasks were valid measures of the same skill (single item reading), we first calculated a Spearman correlation between the scores on the Word and Pseudoword reading subtasks, considering a threshold for a high correlation at $\rho>.80$. Thereafter, a second Spearman correlation between the combined score for Word and Pseudoword reading and the score for Text reading was computed. Our aim with this procedure was to reduce the scores to a single reading measure which gave equal weight to item and to text reading.

\subsubsection{EEG data}

\subsubsection{Data pre-processing}

We used the FieldTrip toolbox (Oostenveld, Fries, Maris, \& Schoffelen, 2011) in Matlab 2014B (Mathworks, Natick, MA) to pre-process and analyse the data. Upon raw data observation, the EEG of three participants at $t_{1}$ was discarded directly due to excessive artifacts ( $n$ for pre-processing $=26$ ), whilst a technical issue during recording prevented us from analysing the data of six 
1 children at $t_{2}(n$ for pre-processing $=26)$. Thereafter, data sets were inspected

2 one by one in order to detect channels with excessive noise and subsequently

3 interpolate them based on the signal of at least three surrounding electrodes

4 (Picton et al., 2000). We then band-pass filtered the data at $0.1-40 \mathrm{~Hz}$ with a

5 two-pass Butterworth filter of order 4 . We had to re-reference the data offline to

6 electrode $\mathrm{Cz}$, since excessive noise in the mastoids forced us to exclude

7 electrodes $\mathrm{A} 1$ and $\mathrm{A} 2$ from further analysis. Such re-referencing approach was

8 used in previous works reporting that $\mathrm{Cz}$ is a suitable reference to explore

9 hemispheric differences (e.g., Picton, 2011; Poelmans, Luts, Vandermosten,

10 Ghesquière, \& Wouters, 2012; Vanvooren, Poelmans, Hofmann, Ghesquiere, \&

11 Wouters, 2014). Next, 19 independent components were estimated from the

12 data using fast ICA (Hyvarinen, 1999). A maximum of 2 independent

13 components per participant -corresponding to heartbeat, eyeblink and eye

14 movements - were identified and removed from the data.

We applied to the audio signal the Hilbert transformation in order to obtain its broadband amplitude envelope (Drullman, Festen, \& Plomp, 1994). Then, the envelope was band-pass filtered through $0.1-40 \mathrm{~Hz}$ and resampled time-locked to EEG signals.

The pre-processed audio and EEG signals were segmented into 2048ms-long epochs with an overlap of 1024-ms (Bortel \& Sovka, 2007;

Bourguignon et al., 2013; Molinaro et al., 2016), an epoch length which results in a frequency resolution of $\sim 0.5 \mathrm{~Hz}$ (i.e., the inverse of the epoch duration). This resolution is actually common in speech-brain coherence analyses (Bourguignon, Baart, Kapnoula, \& Molinaro, 2020; Bourguignon et al., 2013, 2019). Regarding artifact rejection, we discarded epochs with mean amplitude of the whole EEG signal featuring a $z$-score above 3 . Datasets for which more than $30 \%$ of the data had to be discarded under this criterion were not further analysed, reason for which we disposed of the data of one child at $t_{1}$ and two children at $t_{2}$. Therefore, the EEG data of 25 and 24 participants were kept for $t_{1}$ and $t_{2}$, respectively. An average of $93.6 \%(S D=2.8)$ and $95.7 \%(S D=3.3)$ epochs was retained for $t_{1}$ and $t_{2}$, respectively. 


\subsubsection{Coherence to natural speech}

After pre-processing, we used the coherence method described in Halliday et al. (1995) to estimate the dependency between the phase of the EEG signals $(x(t))$ and the phase of speech signal $(y(t))$. This method is a generalization of the Pearson correlation to the frequency domain, since it quantifies the degree of synchronisation between two signals (here $x(t)$ and $y(t)$ ) with a value between 0 and 1 for each frequency bin. As in Pearson correlation, 0 indicates the absence of linear relation, and 1 a perfect linear relation. The 9 formula for the computation of coherence was

0

$$
\operatorname{Coh}_{x y}(f)=\frac{\left|P_{x y}(f)\right|^{2}}{P_{x x}(f) P_{y y}(f)}
$$

with the power- and cross- spectra of $x$ and $y$ defined as

$$
P_{x x}(f)=\frac{1}{N} \sum_{n=1}^{N} X_{n}(f) X_{n}^{*}(f)
$$

$$
P_{y y}(f)=\frac{1}{N} \sum_{n=1}^{N} Y_{n}(f) Y_{n}^{*}(f),
$$

$$
P_{x y}(f)=\frac{1}{N} \sum_{n=1}^{N} X_{n}(f) Y_{n}^{*}(f),
$$

where $N$ is the number of epochs and, $X_{n}(f)$ and $Y_{n}(f)$ denote the Fourier coefficients of the $n^{\text {th }}$ epoch of $x(t)$ and $y(t)$ at frequency bin $f$.

Coherence between the speech envelope and the pre-processed EEG signals was calculated in the $0.1-40-\mathrm{Hz}$ frequency range, which rendered a value for each possible combination of EEG channels, subjects and frequencies. Based on previous studies in adults showing significant coherence

between the speech and the brain signals at $\sim 0.5 \mathrm{~Hz}$ (Bourguignon et al., 2013;

22 Clumeck et al., 2014) and at 4-8 Hz (Ding \& Simon, 2012; Peelle, Gross, \&

23 Davis, 2013), we focused our analysis on these frequency ranges. The 
1 selection of our frequency ranges was supported by the amplitude modulation

2 spectra of our speech stimuli (Figure 1), which showed peaks below $2 \mathrm{~Hz}$ and in between 3 and $7 \mathrm{~Hz}$.

In any case, due to the focus of the present study, here we only report the results for the $0.5 \mathrm{~Hz}$ frequency bin (delta band). For results of the coherence analysis in the theta range $(4-8 \mathrm{~Hz})$ and for a full report of the the 0.5 frequency bin mostly reflects coupling in the $0.1-0.9 \mathrm{~Hz}$ range. This is so because the sensitivity profile of the $0.5 \mathrm{~Hz}$ bin is proportional to the Fourier transform of a 2-s-long boxcar function: $\operatorname{sinc}((f-0.5 \mathrm{~Hz}) / 0.5 \mathrm{~Hz})$ (Destoky et al., 2019).

We used a non-parametric permutation test to estimate group-level statistical significance of speech-brain coherence at the 0.5 frequency bin (Nichols \& Holmes, 2001). First, we calculated coherence between brain signals and the time-flipped speech envelope signal. We then averaged across subjects the coherence values to the genuine and to the flipped speech signals, respectively. A difference value for each electrode was obtained by contrasting the values of the genuine signal against the flipped-signal coherence. We then computed the permutation distribution of the maximum across electrodes of such contrast obtained after having randomly permuted genuine and flipped coherence values within subjects for a subset of 1000 permutations. The $95^{\text {th }}$ percentile of this distribution yielded a significance threshold at $p<0.05$ corrected for multiple comparisons for the initial contrast (Nichols \& Holmes, 2001).

\subsubsection{Joint analysis of reading and EEG data}

First, the EEG and the reading indexes were transformed into z-scores to facilitate comparison of the data. We partialled out the effect of age and IQ from each of our variables of interest before performing any other analysis. In order to do so, we calculated three separate robust linear models with age and IQ at 
$1 \quad t_{1}$ as sole predictors and: i) coherence at $t_{1}$; ii) coherence at $t_{2}$; and iii) reading at

$2 \quad t_{2}$ as dependent variables. The regression was done with the Imrob function

3 from package robustbase in $R$, which uses contemporary algorithms for the estimation of linear regression models in data sets with high variability, as is the case of children data (Maronna, Martin, \& Yohai, 2006). The residuals of the robust linear models were then used for the subsequent Spearman's ranked correlations analyses, which were computed in order to evaluate the relation between speech-brain coherence at $t_{1}$ and $t_{2}$ and reading achievement at $t_{2}$. brain coherence values:

- Raw coherence (Coh): The raw coherence values in the electrodes of interest at $t_{1}$ and $t_{2}$ (see section 3.3 for electrode selection procedure) were used in the correlation analyses. Raw coherence values have been previously used in studies evaluating the relation between coherence and reading abilities (e.g., Power et al., 2016).

- Coherence change index ( $\Delta \mathrm{Coh})$ : We were also interested in examining the putative functional role of the developmental changes in speech-brain coherence from pre-reading into reading stages. To this aim, we computed a coherence change index ( $\Delta \mathrm{Coh})$ from the pre-reading $\left(\mathrm{t}_{1}\right)$ into the reading stage $\left(t_{2}\right)$ as follows:

$$
\Delta \operatorname{Coh}=\operatorname{Coh}\left(t_{2}\right)-\operatorname{Coh}\left(t_{1}\right)
$$

Correlations between the Cls and the reading scores were then computed. The $\Delta$ Coh sign indicates the direction of the change, such that a positive sign means an increase in coherence from $t_{1}$ into $t_{2}$ and a negative sign, a decrease. The aim of this analysis was to check if the change in coherence was a subtler measure to predict reading achievement as compared to raw measures of coherence (see De Vos et al., 2017, for a similar approach).

\subsubsection{Post-hoc analysis: Influence of vocabulary size in the reading-coherence relation}


Firstly, we directly correlated speech-brain coherence with the score in the BEST task to see if our EEG and our vocabulary measure were directly related. Second, we recalculated the partial correlations between low-delta speech-brain coherence and reading controlling for the participants' BEST score in order to examine if the relation between reading and speech-brain coherence was significantly explained by the children's vocabulary size.

\section{Results}

\subsection{Reading tasks}

As a group, participants $(n=31)$ read a mean of 15.1 correct pseudowords per minute $(S D=7.1)$ and a mean of 17.2 correct words per minute $(S D=8.5)$. Within the text, mean number of correct read words was of $20.0(S D=12.0)$. The children's individual scores in the reading tasks can be seen in the supplementary materials (see Table S1 in Supplementary materials).

As expected, the Word reading and the Pseudoword reading subtasks correlated highly $(\rho=.92 ; p<.001)$, and they were hence averaged. Similarly, the correlation coefficient between the Text reading task and the Word and pseudoword reading task was high $(\rho=.83 ; p<.001)$. Therefore, a composite reading measure was created averaging both. Note that the composite measure gave half weight to single item reading and half, to the long text reading. The mean score of the group for the averaged tasks was of 18.1 correct items per minute $(S D=9.4)$. The composite measure was the one used in the subsequent correlational analysis.

\subsection{EEG data}

The EEG data have been described previously (Ríos-López et al., 2020) and will be reported in a summarised manner here. At $t_{1}$, children answered correctly to a mean of $5.1(S D=1.2)$ out of 6 comprehension questions. At $t_{2}$, accuracy increased to $5.6(S D=1.1)$. Descriptively, these high response scores suggested that they were paying attention to the stories (see Ríos-López et al., 
12020 , for detailed results on the relation between speech-brain coherence and 2 intelligibility).

At both testing times, significant coherence to the speech signal was only found in the delta frequency range, and specifically at around $0.5 \mathrm{~Hz}$ (see RíosLópez et al. 2020 for a discussion on the possible reasons for the absence of coherence at other frequency ranges). Accordingly, the $\sim 0.5 \mathrm{~Hz}$ frequency was the only one we inspected further in the correlational analysis between the EEG and the reading data (section 3.3.3).

At $\mathrm{t}_{1}$, electrodes showing significant mean group coherence to speech at $0.5 \mathrm{~Hz}$ were distributed along posterior right $(T 8, \mathrm{P} 8, \mathrm{O} 2)$ and posterior left (T7, $P 7,01$ ) sites of the scalp (see left plot of Figure 2).

Insert Figure 2 around here

At $t_{2}$, we only found significant coherent activity in bilateral temporal electrodes, i.e., in electrodes T7 and T8 (see right plot of Figure 2).

Mean group coherence values in the electrodes showing significant coherence at any of the testing times are shown in Table 1.

Insert Table 1 around here

\subsection{Correlation between EEG and behavioural data}

First, we reduced our electrodes of interest to electrodes that showed significant coherence at both testing times, i.e., to temporal electrodes (T7 and T8). This decision was supported by previous evidence reporting a relation between reading skills and coherence in auditory areas (Hämäläinen et al., 2012; Lizarazu et al., 2015; Molinaro et al., 2016; Power et al., 2016).

Nevertheless, to ensure that the correlations between speech-brain coherence and reading were related to the developmental trajectory of coherence on 
1 temporal electrodes only, this same analysis was performed with parietal

2 electrodes (see Table S2 in Supplementary materials).

\subsubsection{Correlations between coherence and reading output at $t_{2}$}

Coh $\left(\mathrm{t}_{1}\right)$ values in electrode T8 correlated significantly with reading outcome at $\mathrm{t}_{2}(n=25 ; \rho=.52 ; p<.01$; False discovery rate [FDR]- $p=.05)$, such that children with larger coherence values in this electrode at $t_{1}$ showed better reading performance at $t_{2}$ (see Figure 3 ). Such relation was not found for the Coh $\left(\mathrm{t}_{1}\right)$ values in electrode T7 $(n=25 ; \rho=.24 ; p=.25)$. Finally, Coh $\left(\mathrm{t}_{2}\right)$ values did not correlate with reading outcome in any of the electrodes $(n=24 ; \mathrm{T} 7: \rho=$ $.15 ; p=.48 ;$ T8: $\rho=-.08 ; p=.71$ ).

Insert Figure 3 around here

\subsubsection{Correlations $\Delta$ Coh from $t_{1}$ to $t_{2}$ and reading output at $t_{2}$}

The $\Delta$ Coh from $t_{1}$ to $t_{2}$ in electrode $T 8$ correlated negatively with reading achievement in $\mathrm{t}_{2}(n=20 ; \rho=-.44 ; p=.05)$, such that children with larger increase in coherence in electrode T8 showed poorer reading performance at $\mathrm{t}_{2}$ (see Figure 3). Note that this correlation was not significant after FDR correction (FDR- $p=.10)$. No significant correlation between the change in coherence from $\mathrm{t}_{1}$ to $\mathrm{t}_{2}$ and reading achievement at $\mathrm{t}_{2}$ was found for electrode T7 $(n=20 ; \rho=-$ $.13 ; p=.58)$.

1.6.3. Results of the post-hoc analyses: The impact of general linguistic knowledge in the reading-coherence relation

As mentioned above, our post-hoc analyses including the picture-naming task served the purpose of testing if the relation between speech-brain coherence and reading was mediated by a more general linguistic measure such as vocabulary size. Firstly, none of the Spearman correlations between speech-brain coherence and the score in the vocabulary task was significant (all $\rho s<.28$; all uncorrected $p s>.07$ ), suggesting that, with the methods used, these measures were not directly related. Secondly, the partial correlation 
1 between raw speech-brain coherence at $\mathrm{t} 1$ and reading at $\mathrm{t} 2$ controlling for

2 vocabulary at $\mathrm{t} 1$ was still significant $(n=25 ; \rho=.48 ; p<.01)$, suggesting that the

3 relation between speech-brain coherence and reading was not (at least significantly) mediated by general non-phonological language knowledge at $\mathrm{t} 1$.

\section{Discussion}

The aim of this study was to establish a longitudinal relation between reading acquisition and neural oscillations responding to natural speech at low delta frequencies $(<1 \mathrm{~Hz})$. Analysing the coherence phenomenon from different perspectives (raw coherence and change index) led to complementary results that will help characterising the value of the coherence measure in predicting future reading achievement. Furthermore, although our sample size was modest, we were able to detect interesting relations that should be explored in depth in future studies with larger samples.

Raw coherence values in the right hemisphere at $t_{1}$ predicted reading achievement at $t_{2}$, such that children with larger coherence values in electrode T8 prior to formal reading instruction (before starting Grade 1) were the ones with better reading scores after finishing Grade 1. This result is in line with previous theories and cross-sectional results in the dyslexia literature suggesting the existence of a tight relation between right-hemisphere coherence to the delta rhythms of speech and reading achievement (Goswami, 2011, 2018, Lallier et al., 2018, 2017; Molinaro et al., 2016). According to these accounts of dyslexia, specialised right-hemispheric coherence to the lowfrequency modulations of speech might be crucial in order to further process fast occurring phonemic information, which might be processed preferentially in the left hemisphere or bilaterally (Boemio, Fromm, Braun, \& Poeppel, 2005; Bourguignon et al., 2013; Gross et al., 2013).

To our knowledge, this is the first study reporting a longitudinal relation between pre-reading coherence to natural speech and future reading achievement in typically developing children. None of the children in the sample was at family risk of developing dyslexia or any other speech pathology. According to our data, the speech-brain coherence measure was even able to 
1 capture longitudinal subtle reading variance in non-at risk samples, where

2 variability is reduced as compared to pathological populations.

By contrast, we did not find any correlation between low delta band speech-brain coherence and reading outcome one year after reading instruction had started (i.e., at $t_{2}$ ). Thus, our overall results fit only partially within the postulates of the temporal sampling framework (Goswami, 2011) and are at odds with previous cross-sectional evidence reporting a relation between reading skills and delta band coherence in response to auditory stimuli in dyslexic participants (Hämäläinen et al., 2012; Molinaro et al., 2016; Power et al., 2016). Again, a crucial difference between our and previous studies is the fact that our sample consisted exclusively of typically developing children who were learning to read, whereas their samples included pre-adolescent and/or adult dyslexic readers. Indeed, the Molinaro et al. (2016) study found a positive correlation between phonology and connectivity patterns from the right auditory cortex to the inferior fontal gyrus only in the group of dyslexic participants, whereas such association was not present in the control group.

Overall, our findings have no precedent in the literature, and we believe that the change in the predictive power of neural coherence from pre-reading into reading stages found in our sample could shed light on the changing nature of the relation between neural rhythmic sensitivity and reading development. Indeed, our results suggest that the power of rhythmic entrainment to predict reading outcome might be restricted to very early stages of typical development and lose strength as the children acquire further linguistic skills that influence reading performance (see Nagy, Carlisle, \& Goodwin, 2014 for a review). Note that, for example, morphological knowledge has been proven to be an important predictor of reading skills across the school years (Berninger, Abbott, Nagy, \& Carlisle, 2010), while the contribution of phonological awareness (and hence rhythm) could be restricted to very early stages of reading development (Nagy, Berninger, \& Abbott, 2006). Reading acquisition in consistent orthographies such as the Basque one develops fast as compared to opaque orthographies such as the English one (Seymour, Aro, \& Erskine, 2003). In consequence, it is possible that our children were already past the alphabetic phase, based mainly 
1 on the implementation of letter-sound correspondences, and into a more

2 advanced reading phase in which they already relied on morphological/lexical

3 knowledge (Frith, 1986; Marsh, Friedman, Welch, \& Desberg, 1981; Nagy et al.,

4 2014). If this were the case, it is not surprising that neural rhythmic entrainment

5 did not predict reading at the later stage since, according for example to the

6 temporal sampling theory (Goswami, 2011), rhythmic sensibility would be core

7 in the formation of phonological representations, and the quality of these

8 representations is most likely essential during very early (decoding) stages as

9 opposed to more advanced reading stages, at least for typical reading development (Nagy et al., 2006). In this line, cross-linguistic studies have shown that phonological awareness contributes more strongly at the early phases of reading acquisition, a contribution that would later vanish (Vaessen et al., 2010). It is hence acceptable to hypothesise that the rhythm-reading relation could change as a function of the phonological demands required in the specific moment at the reading acquisition course (Lundetræ \& Thomson, 2017). Once children are past the decoding phase, the contribution of early perceptual indexes such as neural rhythmic sensitivity to reading variability might be modest, or already non-existent. For all this, we are inclined to believe that the influence of rhythmic sensibility might be more visible at very initial stages of formal reading instruction, at least in transparent orthographies. This hypothesis might be explored in future (ideally cross-linguistic) studies.

Crucially, the changing role of delta coherence in different stages of reading acquisition was supported by our developmental analysis, which showed that the increase in coherence ( $\Delta \mathrm{Coh})$ in electrode T8 from $t_{1}$ to $t_{2}$ correlated negatively with reading achievement. Although this result was not significant after correction for multiple comparisons, we tend to believe that this was a power issue derived from our modest sample size and that it still deserves further attention. The fact that children with larger increase in coherence in electrode T8 during initial formal reading instruction were the ones with poorer reading outcomes admits at least two interpretations.

First, it could suggest that the increase in coherence between the testing times might be a compensatory mechanism elicited by reading instruction itself. 
1 Note that this was the interpretation favoured by a previous longitudinal study

2 showing that after reading instruction, dyslexic children showed a larger

3 increase in beta band power in response to $20-\mathrm{Hz}$ AM signals as compared to control children (De Vos et al., 2017).

Nevertheless, we are inclined to support another interpretation of the developmental results which fits better with the results of the individual testing times, that is, with our finding of a significant correlation between pre-reading speech-brain coherence $\left(a t_{1}\right)$ and later reading performance $\left(a t t_{2}\right)$, whereas speech-brain coherence was not associated with reading performance within $t_{2}$. Indeed, what our results seem to indicate is that a high speech-brain coherence at low delta frequencies prior to reading acquisition could be the driving force for reading acquisition, whilst reading instruction itself could increase speech-brain coherence in children with low pre-reading delta tracking. Our interpretation of the increase in coherence as a compensatory mechanism is in line with the one provided by De Vos et al. (2017). Namely, in children with lower pre-reading levels of speech-brain coherence, reading instruction could provide a boost that brings speech-brain coherence to the level of that of more successful readers whose pre-reading coherence level was already high (and predictive of reading performance). In any case, no significant relation between coherence and reading within $t_{2}$ was found, suggesting again that, at least in children who are not at family risk of developing dyslexia (but who could nevertheless develop reading problems later), coherence might be a valuable predictor of reading performance especially before reading instruction occurs.

Also, we believe it is necessary to briefly discuss the nature of the reading-delta entrainment relation. Indeed, delta band entrainment has been proposed to reflect not only acoustic parsing of the signal, but also higher-order level processes related to, for example, syntactic processing (Ding, Melloni, Zhang, Tian, \& Poeppel, 2016; Keitel, Gross, \& Kayser, 2017; Meyer, Henry, Gaston, Schmuck, \& Friederici, 2016; Molinaro \& Lizarazu, 2018). Given our paradigm, we cannot conclude if the relation between coherence to speech at the $0.5 \mathrm{~Hz}$ is purely auditory according to what the temporal sampling framework suggests (Goswami, 2011) or mediated by higher processes 
related to language comprehension (see Meyer, 2017 for a discussion of this topic). Future studies comparing stimuli with and without linguistic content are necessary to disentangle the specifics of this relation.

In the same vein, our data cannot disentangle the extent to which the relation between speech-brain entrainment and reading is mediated by different sets of language processing skills. Our study relies on the hypothesis that reading development's foundations are built on the acquisition of phonological skills (Bishop \& Snowling, 2004; Melby-Lervåg, Lyster, \& Hulme, 2012, for a review), which have been linked to the integrity of speech-brain synchronization mechanisms (Destoky et al., 2020; Di Liberto et al., 2018; Goswami, 2011, 2017; Molinaro et al., 2016; Power et al., 2016). However, general language knowledge - or non-phonological language skills, such as vocabulary, morphosyntax, or discourse skills - is likely to exert some influence on the development of these phonological skills, and therefore on reading skills later one. For example, it has been shown that the amount of exposure to a language (an index of general language knowledge and experience) contributes to the development of phonological abilities (Garcia-Sierra et al., 2011; for a review, see Nittrouer, 2002). In addition, non-phonological language factors such as vocabulary mediate the progressive acquisition of refined phonological abilities (Gathercole et al., 1991; Parra et al., 2011) that will support reading acquisition later on. Therefore, it is reasonable to assume that general nonphonological language knowledge will contribute to speech-brain synchronization, too. In order to partially test these hypotheses, we examined the putative mediation role of vocabulary size in the reading-coherence relation. Our analyses of the picture naming suggest that the variance shared between reading skills and speech-brain coherence may be partly independent from the variance shared between general language knowledge and speech-brain coherence. Future studies will thrive on investigating the interplay between the variance shared by speech-brain coherence and phonological language factors in the one hand, and non-phonological language knowledge (e.g., vocabulary, syntax) in the other hand, in order to understand better how reading develops. 
Overall, our study adds up to an increasing list of scientific evidence

2 reporting a tight relation between reading skills and coherence to natural speech and/or language-relevant amplitude modulations in older children and adults (Abrams et al., 2009; Hämäläinen et al., 2012; Lizarazu et al., 2015; Molinaro et al., 2016; Power et al., 2016, 2013). Together with previous evidence reporting a relation between behavioural markers of the sensitivity to linguistic rhythm and pre-reading skills such as letter name knowledge (Ríos López, Molinaro, \& Lallier, 2019) or phonological awareness and rapid automatized naming (Carr, White-Schwoch, Tierney, Strait, \& Kraus, 2014) our results suggest that the combination of both behavioural and (right hemisphere) neural indexes of rhythm processing could inform the early detection and prognosis of developmental reading disorders.

Lastly, the present study has some limitations. First, since speech-brain coherence had never been tested in very young children, we settled for a rather small sample size as a first approach to the field in the hope that future studies with more statistical power will shed light upon the questions addressed in the current work. Although we were able to establish a preliminary link between prereading coherence and later reading, the possible mediators of such relationship remain understudied. Theories such as the TSF suggest that impaired speech-brain coherence would lead to (pre-reading) phonological impairments which would in turn derive in reading disorders (Goswami, 2011). Our modest sample size did not allow us to fit more sophisticated models (e.g., path analysis) including other behavioural pre-reading factors (e.g., RAN, letter name knowledge, phonological awareness, etc.); in our case, this would have derived in a poor observations-to-parameters ratio. We hope future studies with larger sample sizes can shed light on this relevant matter.

Also, it is important to note that our participants were Basque-Spanish bilinguals, and that previous studies have reported differences in language activation between monolinguals and bilinguals (e.g., Hull \& Vaid, 2007). In order to discard possible effects of the children's language dominance in our results, we computed separate pair-wise correlations between language dominance as measured by the BEST battery (de Bruin et al., 2017) and the 
1 behavioural and EEG tasks used across this research work. The results showed

2 that none of the tasks correlated significantly with the Language dominance

3 measure (all $\rho$ s $<.15 ; p s>.20$ ). Despite this, we cannot fully discard that our

4 findings regarding brain coherence, and especially the consistent bilateral

5 pattern of activation, has something to do with the psycholinguistic profile of our

6 participants (see Ríos-López et al., 2020). Furthermore, we used different

7 stories for $\mathrm{t} 1$ and $\mathrm{t} 2$. This was done in order to ensure comparability within each

8 of the testing times. Although the syllabic rhythm of the different linguistic

9 signals was comparable, the use of different stimuli could have an impact upon speech-brain coherence. Larger longitudinal studies might want to control for this factor by counterbalancing the stories across the years. Finally, due to the young age of our participants at the first testing time, we used EEG, a technique with high temporal resolution but with important spatial limitations as compared to other techniques such as MEG. Our conclusions on the topography of our effects are hence limited at the level of the scalp, with the evident limitations that this carries along. Future studies will hopefully deal with this issue.

\section{Conclusions}

\section{Acknowledgments}


1 The authors wish to thank the children and their parents for taking part in this

2 study, and Alexia Antzaka and José Pérez-Navarro for their help with the EEG

3 testing.

4 Funding information

5 This work was supported by the Spanish Ministry of Economy, Industry and

6 Competitiveness [grant PSI2015-6533-P], the Basque Government [grant

7 PI_2016_1_0014] and the Gipuzkoako Foru Aldundia [grant EXP. 99/17] to

$8 \quad$ N.M.; the Spanish Ministry for Science, Innovation and Universities [grants

9 RTI2018-096242-B-I00 and RYC-2015-17356] to M.L; and the Basque

10 Government (BERC 2018-2021 program) and the Severo Ochoa Program

11 [grant SEV-2015-049] to the BCBL.

\section{Declaration of interests}

The authors declare that they have no known competing financial interests or personal relationships that could have appeared to influence the work reported

16 in this paper.

\section{CRediT author statement}

Paula Ríos-López: Conceptualization, Methodology, Software, Formal analysis, Investigation, Writing - Original Draft, Visualization. Nicola Molinaro: Conceptualization, Methodology, Writing - Reviewing and editing, Supervision, Funding Acquisition. Mathieu Bourguignon: Software, Formal analysis, Writing

23 - Reviewing and editing. Marie Lallier: Conceptualization, Methodology, 24 Writing - Reviewing and editing, Supervision, Project administration, Funding Acquisition. 
The data that support the findings of this study are publicly available in the Open Science Framework (OSF) repository (https://osf.io/vpagr/).

DOI: 10.17605/OSF.IO/VPAGR 


\section{References}

Abrams, D. A, Nicol, T., Zecker, S., \& Kraus, N. (2009). Abnormal cortical processing of the syllable rate of speech in poor readers. Journal of Neuroscience, 29(24), 7686-7693. https://doi.org/10.1523/JNEUROSCI.5242-08.2009

Alexandrou, A. M., Saarinen, T., Kujala, J., \& Salmelin, R. (2018). Cortical entrainment: what we can learn from studying naturalistic speech perception. Language, Cognition and Neuroscience, 1-13. https://doi.org/10.1080/23273798.2018.1518534

Anthony, J. L., \& Francis, D. J. (2005). Development of phonological awareness. Current Directions in Psychological Science, 14(5), 255-259. https://doi.org/10.1111/j.0963-7214.2005.00376.x

Berninger, V. W., Abbott, R. D., Nagy, W., \& Carlisle, J. (2010). Growth in phonological, orthographic, and morphological awareness in grades 1 to 6 . Journal of Psycholinguistic Research, 39(2), 141-163. https://doi.org/10.1007/s10936-009-9130-6

Bishop, D. V., \& Snowling, M. J. (2004). Developmental dyslexia and specific language impairment: Same or different?. Psychological bulletin, 130(6), 858.

Boemio, A., Fromm, S., Braun, A., \& Poeppel, D. (2005). Hierarchical and asymmetric temporal sensitivity in human auditory cortices. Nature Neuroscience, 8(3), 389-395. https://doi.org/10.1038/nn1409

Bortel, R., \& Sovka, P. (2007). Approximation of statistical distribution of magnitude squared coherence estimated with segment overlapping. Signal Processing, 87(5), 1100-1117. https://doi.org/10.1016/j.sigpro.2006.10.003

Bourguignon, M., Baart, M., Kapnoula, E. C., \& Molinaro, N. (2020). Lip-reading enables the brain to synthesize auditory features of unknown silent speech. Journal of Neuroscience, 40(5), 1053-1065. 
2 Bourguignon, M., De Tiège, X., De Beeck, M. O., Ligot, N., Paquier, P., Van

\section{https://doi.org/10.1523/JNEUROSCI.1101-19.2019} Bogaert, P., ... Jousmäki, V. (2013). The pace of prosodic phrasing couples the listener's cortex to the reader's voice. Human Brain Mapping, 34(January 2012), 314-326. https://doi.org/10.1002/hbm.21442

Bourguignon, M., Molinaro, N., Lizarazu, M., Taulu, S., Jousmäki, V., Lallier, M., ... Tiège, X. De. (2019). Neocortical activity tracks syllable and phrasal structure of self-produced speech during reading aloud. BioRxiv, 744151. https://doi.org/10.1101/744151

Carr, K. W., White-Schwoch, T., Tierney, A. T., Strait, D. L., \& Kraus, N. (2014). Beat synchronization predicts neural speech encoding and reading readiness in preschoolers. Proceedings of the National Academy of Science, 111(40), 14559-14564. https://doi.org/10.1073/pnas.1406219111

De Bruin, A., Carreiras, M., \& Duñabeitia, J. A. (2017). The BEST dataset of language proficiency. Frontiers in Psychology, 8, 522. https://doi.org/10.3389/fpsyg.2017.00522

De Vos, A., Vanvooren, S., Vanderauwera, J., Ghesquière, P., \& Wouters, J. (2017). A longitudinal study investigating neural processing of speech envelope modulation rates in children with (a family risk for) dyslexia. Cortex, 93, 206-219. https://doi.org/10.1016/j.cortex.2017.05.007

Destoky, F., Bertels, J., Niesen, M., Wens, V., Vander Ghinst, M., Leybaert, J., ... \& Bourguignon, M. (2020). Cortical tracking of speech in noise accounts for reading strategies in children. PLoS biology, 18(8), e3000840. https://doi.org/10.1371/journal.pbio.3000840

Destoky, F., Philippe, M., Bertels, J., Verhasselt, M., Coquelet, N., Vander Ghinst, M., ... Bourguignon, M. (2019). Comparing the potential of MEG and EEG to uncover brain tracking of speech temporal envelope. Neurolmage, 184, 201-213. 
https://doi.org/10.1016/j.neuroimage.2018.09.006

Di Liberto, G. M., Peter, V., Kalashnikova, M., Goswami, U., Burnham, D., \& Lalor, E. C. (2018). Atypical cortical entrainment to speech in the right hemisphere underpins phonemic deficits in dyslexia. Neuroimage, 175, 7079. https://doi.org/10.1016/j.neuroimage.2018.03.072

Ding, N., Melloni, L., Zhang, H., Tian, X., \& Poeppel, D. (2016). Cortical tracking of hierarchical linguistic structures in connected speech. Nature Neuroscience, 19(1), 158-164. https://doi.org/10.1038/nn.4186

Ding, N., \& Simon, J. Z. (2012b). Neural coding of continuous speech in auditory cortex during monaural and dichotic listening. Journal of Neurophysiology, 107(October 2011), 78-89. https://doi.org/10.1152/jn.00297.2011

Drullman, R., Festen, J. M., \& Plomp, R. (1994). Effect of temporal envelope smearing on speech reception. The Journal of the Acoustical Society of America, 95(2), 1053-1064. https://doi.org/10.1121/1.408467

Frith, U. (1986). A developmental framework for developmental dyslexia. Annals of Dyslexia, 36(1), 67-81. https://doi.org/10.1007/BF02648022

Goswami, U. (2011). A temporal sampling framework for developmental dyslexia. Trends in Cognitive Sciences, 15(1), 3-10. https://doi.org/10.1016/j.tics.2010.10.001

Goswami, U. (2018). A neural basis for phonological awareness? An oscillatory temporal-sampling perspective. Current Directions in Psychological Science, 27(1), 56-63. https://doi.org/10.1177/0963721417727520

Gross, J., Hoogenboom, N., Thut, G., Schyns, P., Panzeri, S., Belin, P., \& Garrod, S. (2013). Speech rhythms and multiplexed oscillatory sensory coding in the human brain. PLoS Biology, 11(12), e1001752. https://doi.org/10.1371/journal.pbio.1001752 
1 Halliday, D., Rosenberg, J., Amjad, A., Breeze, P., Conway, B., \& Farmer, S. (1995). A framework for the analysis of mixed time series/point process data - theory and application to the study of physiological tremor, single motor unit discharges and electromyograms. Progress in Biophysics and Molecular Biology, 64(2-3), 237-278. https://doi.org/10.1016/S00796107(96)00009-0

Hyvarinen, A. (1999). Fast and robust fixed-point algorithms for independent component analysis. IEEE transactions on Neural Networks, 10(3), 626634. https://doi.org/10.1109/72.761722

Hämäläinen, J. A., Rupp, A., Soltész, F., Szücs, D., \& Goswami, U. (2012). Reduced phase locking to slow amplitude modulation in adults with dyslexia: An MEG study. Neurolmage, 59(3), 2952-2961. https://doi.org/10.1016/j.neuroimage.2011.09.075

Hull, R., \& Vaid, J. (2007). Bilingual language lateralization: A meta-analytic tale of two hemispheres. Neuropsychologia, 45(9), 1987-2008. https://doi.org/10.1016/J.NEUROPSYCHOLOGIA.2007.03.002

Keitel, A., Gross, J., \& Kayser, C. (2017). Speech tracking in auditory and motor regions reflects distinct linguistic features. bioRxiv, 195941. https://doi.org/10.1101/195941

Keitel, A., Gross, J., \& Kayser, C. (2018). Perceptually relevant speech tracking in auditory and motor cortex reflects distinct linguistic features. PLoS Biology, 16(3), 1-19. https://doi.org/10.1371/journal.pbio.2004473

Kösem, A., Bosker, H. R., Takashima, A., Meyer, A., Jensen, O., \& Hagoort, P. (2018). Neural Entrainment Determines the Words We Hear. Current Biology, 28(18), 2867-2875.e3. https://doi.org/10.1016/j.cub.2018.07.023

Lallier, M., Lizarazu, M., Molinaro, N., Bourguignon, M., Ríos-López, P., \& Carreiras, M. (2018). From auditory rhythm processing to grapheme-tophoneme conversion: How neural oscillations can shed light on 
Lallier, M., Molinaro, N., Lizarazu, M., Bourguignon, M., \& Carreiras, M. (2017). Amodal atypical neural oscillatory activity in dyslexia. Clinical Psychological Science, 5(2), 379-401. https://doi.org/10.1177/2167702616670119

Lallier, M., Valdois, S., Lassus-Sangosse, D., Prado, C., \& Kandel, S. (2014). Impact of orthographic transparency on typical and atypical reading development: Evidence in French-Spanish bilingual children. Research in Developmental Disabilities, 35(5), 1177-1190. https://doi.org/10.1016/j.ridd.2014.01.021

Lizarazu, M., Lallier, M., Molinaro, N., Bourguignon, M., Paz-Alonso, P. M., Lerma-Usabiaga, G., \& Carreiras, M. (2015). Developmental evaluation of atypical auditory sampling in dyslexia: Functional and structural evidence. Human Brain Mapping, 36(12), 4986-5002. https://doi.org/10.1002/hbm.22986

Lundetræ, K., \& Thomson, J. M. (2017). Rhythm production at school entry as a predictor of poor reading and spelling at the end of first grade. Reading and Writing, 31(1), 215-237. https://doi.org/10.1007/s11145-017-9782-9

Maronna, R. A. R. D., Martin, R. D., \& Yohai, V. (2006). Robust statistics (Vol. 1). Chichester: John Wiley \& Sons Inc. https://doi.org/10.1002/0470010940

Marsh, G., Friedman, M., Welch, V., \& Desberg, P. (1981). A cognitivedevelopmental theory of reading acquisition. Reading research: Advances in theory and practice, 3, 199-221.

Melby-Lervåg, M., Lyster, S. A. H., \& Hulme, C. (2012). Phonological skills and their role in learning to read: a meta-analytic review. Psychological bulletin, 138(2), 322. https://doi.org/10.1037/a0026744 
1 Meyer, L. (2017). The neural oscillations of speech processing and language comprehension: State of the art and emerging mechanisms. European Journal of Neuroscience, 13748. https://doi.org/10.1111/ejn.13748

Meyer, L., Henry, M. J., Gaston, P., Schmuck, N., \& Friederici, A. D. (2016). Linguistic bias modulates interpretation of speech via neural delta-band oscillations. Cerebral Cortex, 27(9), 4293-4302. https://doi.org/10.1093/cercor/bhw228

Molinaro, N., \& Lizarazu, M. (2018). Delta (but not theta)-band cortical entrainment involves speech-specific processing. European Journal of Neuroscience, 13811. https://doi.org/10.1111/ejn.13811

Molinaro, N., Lizarazu, M., Lallier, M., Bourguignon, M., \& Carreiras, M. (2016). Out-of-synchrony speech entrainment in developmental dyslexia. Human Brain Mapping, 37(8), 2767-2783. https://doi.org/10.1002/hbm.23206

Nagy, W. E., Berninger, V. W., \& Abbott, R. D. (2006). Contributions of morphology beyond phonology to literacy outcomes of upper elementary and middle-school students. Journal of Educational Psychology, 98(1), 134-147. https://doi.org/10.1037/0022-0663.98.1.134

Nagy, W. E., Carlisle, J. F., \& Goodwin, A. P. (2014). Morphological knowledge and literacy acquisition. Journal of Learning Disabilities, 47(1), 3-12. https://doi.org/10.1177/0022219413509967

Nichols, T. E., \& Holmes, A. P. (2001). Nonparametric permutation tests for functional neuroimaging: A primer with examples. Human Brain Mapping, 15(1), 1-25. https://doi.org/10.1002/hbm.1058

Oostenveld, R., Fries, P., Maris, E., \& Schoffelen, J.-M. (2011). FieldTrip: Open source software for advanced analysis of MEG, EEG, and invasive electrophysiological data. Computational Intelligence and Neuroscience, 2011. https://doi.org/10.1155/2011/156869 
1 Peelle, J. E., Gross, J., \& Davis, M. H. (2013). Phase-locked responses to speech in human auditory cortex are enhanced during comprehension. Cerebral Cortex, 23(6), 1378-1387. https://doi.org/10.1093/cercor/bhs118

Peirce, J. W. (2009). Generating stimuli for neuroscience using PsychoPy. Frontiers in Neuroinformatics, 2, 10. https://doi.org/10.3389/neuro.11.010.2008

Picton, T. (2011). Human auditory evoked potentials. Ear and Hearing. Plural Publishing. https://doi.org/10.1097/AUD.0b013e3182498db9

Picton, T. W., Bentin, S., Berg, P., Donchin, E., Hillyard, S. A., Johnson, R., ... Taylor, M. J. (2000). Guidelines for using human event-related potentials to study cognition: Recording standards and publication criteria.

Poelmans, H., Luts, H., Vandermosten, M., Ghesquière, P., \& Wouters, J. (2012). Hemispheric asymmetry of auditory steady-state responses to monaural and diotic stimulation. Journal of the Association for Research in Otolaryngology, 13(6), 867-876. https://doi.org/10.1007/s10162-012-0348$\mathrm{x}$

Poeppel, D. (2003). The analysis of speech in different temporal integration windows: Cerebral lateralization as "asymmetric sampling in time." Speech Communication, 41, 245-255. https://doi.org/10.1016/S01676393(02)00107-3

Poeppel, D., Idsardi, W. J., \& Van Wassenhove, V. (2008). Speech perception at the interface of neurobiology and linguistics. Philosophical Transactions of the Royal Society of London B: Biological Sciences, 363(1493), 10711086. https://doi.org/10.1098/rstb.2007.2160

Poeppel, D., \& Assaneo, M. F. (2020). Speech rhythms and their neural foundations. Nature Reviews Neuroscience. Nature Research. https://doi.org/10.1038/s41583-020-0304-4

Power, A. J., Colling, L. J., Mead, N., Barnes, L., \& Goswami, U. (2016). Neural 
encoding of the speech envelope by children with developmental dyslexia. Brain and Language, 160, 1-10. https://doi.org/10.1016/j.bandl.2016.06.006

Power, A. J., Mead, N., Barnes, L., \& Goswami, U. (2013). Neural entrainment to rhythmic speech in children with developmental dyslexia. Frontiers in Human Neuroscience, 7(November), 777. https://doi.org/10.3389/fnhum.2013.00777

Ríos-López, P., Molinaro, N., Bourguignon, M., \& Lallier, M. (2020).

Development of neural oscillatory activity in response to speech in children from 4 to 6 years old. Developmental Science, 1-16. https://doi.org/10.1111/desc.12947

Ríos-López, P., Molinaro, N., \& Lallier, M. (2019). Tapping to a beat in synchrony predicts brain print sensitivity in pre-readers. Brain and language, 199, 104693. https://doi.org/10.1016/j.bandl.2019.104693

Ríos-López, P., Molnar, M. T., Lizarazu, M., \& Lallier, M. (2017). The role of slow speech amplitude envelope for speech processing and reading development. Frontiers in Psychology, 8(August), 1497. https://doi.org/10.3389/FPSYG.2017.01497

Seymour, P. H. K., Aro, M., \& Erskine, J. M. (2003). Foundation literacy acquisition in European orthographies. British Journal of Psychology, 94(2), 143-174. https://doi.org/10.1348/000712603321661859

Vaessen, A., Bertrand, D., Tóth, D., Csépe, V., Faísca, L., Reis, A., \& Blomert, L. (2010). Cognitive development of fluent word reading does not qualitatively differ between transparent and opaque orthographies. Journal of Educational Psychology, 102(4), 827-842. https://doi.org/10.1037/a0019465

Vanvooren, S., Poelmans, H., Hofmann, M., Ghesquiere, P., \& Wouters, J. (2014). Hemispheric asymmetry in auditory processing of speech envelope 
1 modulations in prereading children. Journal of Neuroscience, 34(4), 1523-

2 1529. https://doi.org/10.1523/JNEUROSCI.3209-13.2014

3 Wechsler, D. (2002). WPPSI-III administration and scoring manual. Psychological Corporation.

5 Wood, C., Wade-Woolley, L., \& Holliman, A. J. (2009). Phonological awareness:

6 Beyond phonemes. In C. Wood \& V. Connelly (Eds.), Contemporary

7 perspectives on reading and spelling (pp. 7-23). New York: Routledge.

8 Ziegler, J. C., \& Goswami, U. (2005). Reading acquisition, developmental dyslexia, and skilled reading across languages: a psycholinguistic grain

10 size theory. Psychological Bulletin, 131(1), 3-29.

$11 \quad$ https://doi.org/10.1037/0033-2909.131.1.3 
Table 1. Group-level coherence values at $0.5 \mathrm{~Hz}$. Electrodes that showed significant coherence are marked with an asterisk $\left({ }^{*}\right)$.

\begin{tabular}{|c|c|c|c|c|c|c|c|c|c|c|c|c|}
\hline & \multicolumn{8}{|c|}{ Electrode } & & & & \\
\hline & \multicolumn{2}{|c|}{ T7 } & \multicolumn{2}{|c|}{ P7 } & \multicolumn{2}{|c|}{ T8 } & \multicolumn{2}{|c|}{ P8 } & \multicolumn{2}{|c|}{01} & \multicolumn{2}{|c|}{02} \\
\hline TT & $t_{1}$ & $t_{2}$ & $t_{1}$ & $t_{2}$ & $t_{1}$ & $t_{2}$ & $t_{1}$ & $t_{2}$ & $t_{1}$ & $t_{2}$ & $t_{1}$ & $t_{2}$ \\
\hline$M$ & $.015^{\star}$ & $.019^{*}$ & $.013^{*}$ & .013 & $.016^{\star}$ & $.020^{*}$ & $.014^{*}$ & .012 & $.010^{*}$ & .009 & $.011^{*}$ & .009 \\
\hline$S D$ & .026 & .027 & .016 & .013 & .019 & .021 & .022 & .011 & .009 & .007 & .012 & .012 \\
\hline
\end{tabular}



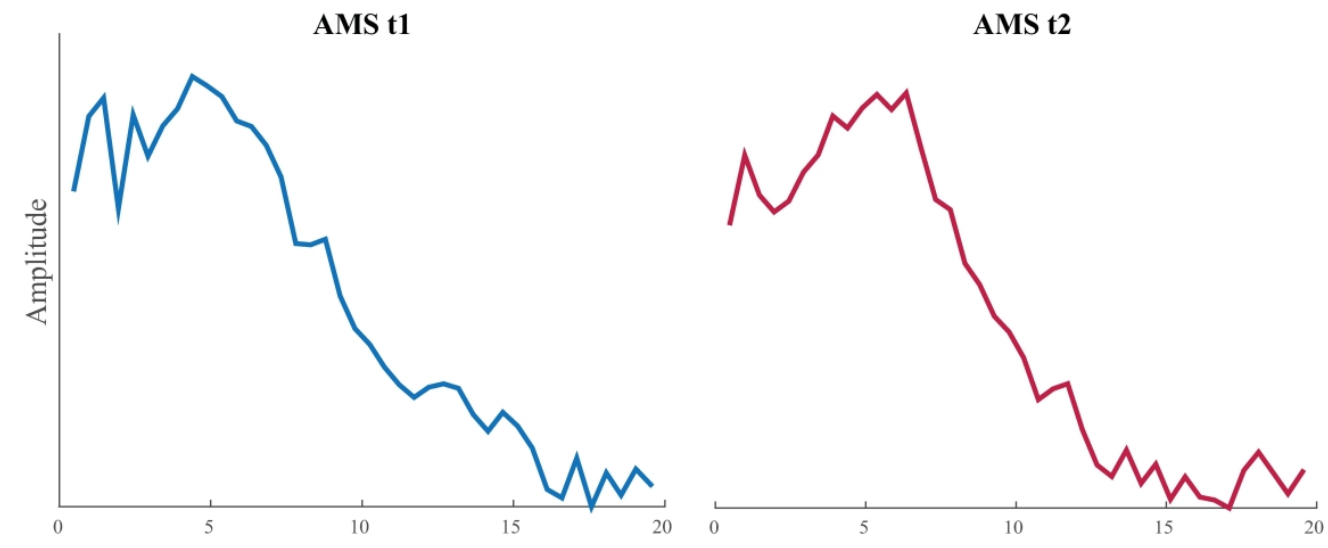

Frequency in $\mathrm{Hz}$

Figure 1. Amplitude modulation spectra of the stimuli at $t 1$ (top panel) and $t 2$ (bottom panel). Amplitude values were multiplied by $f$ to compensate for the classical $1 / f$ trend (Poeppel \& Assaneo, 2020). This increases the visibility of the fluctuations around the trend.

\section{$245 \times 111 \mathrm{~mm}(600 \times 600 \mathrm{DPI})$}



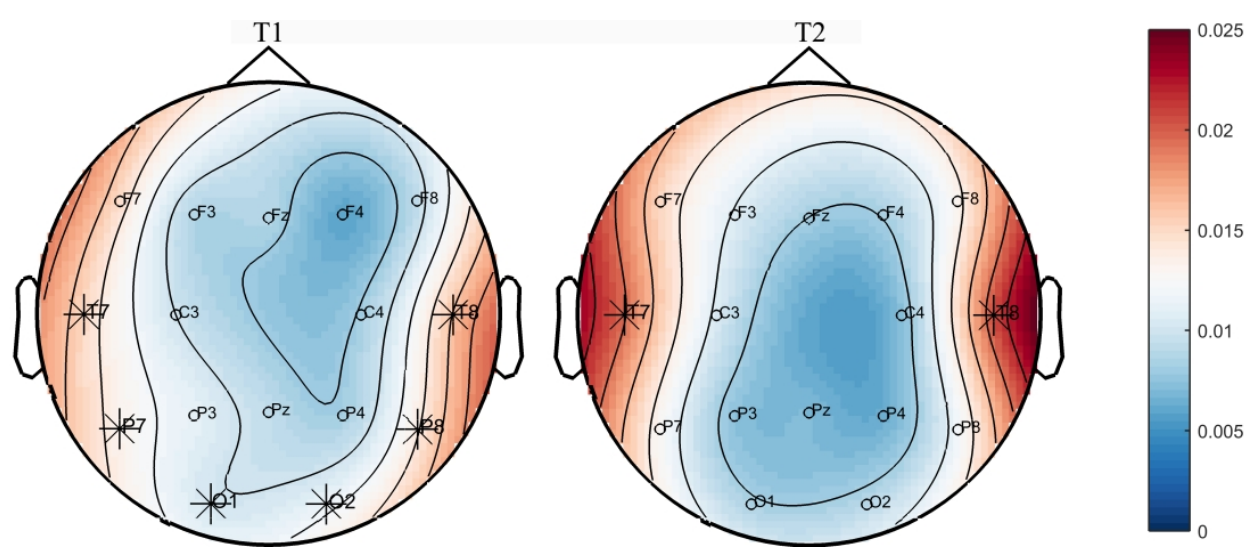

Figure 2. Coherence maps at the $0.5 \mathrm{~Hz}$ frequency band across the testing times. Electrodes that showed significant coherence to speech are marked with an asterisk (*). Reproduced with permission from RíosLópez et al. (2020). 

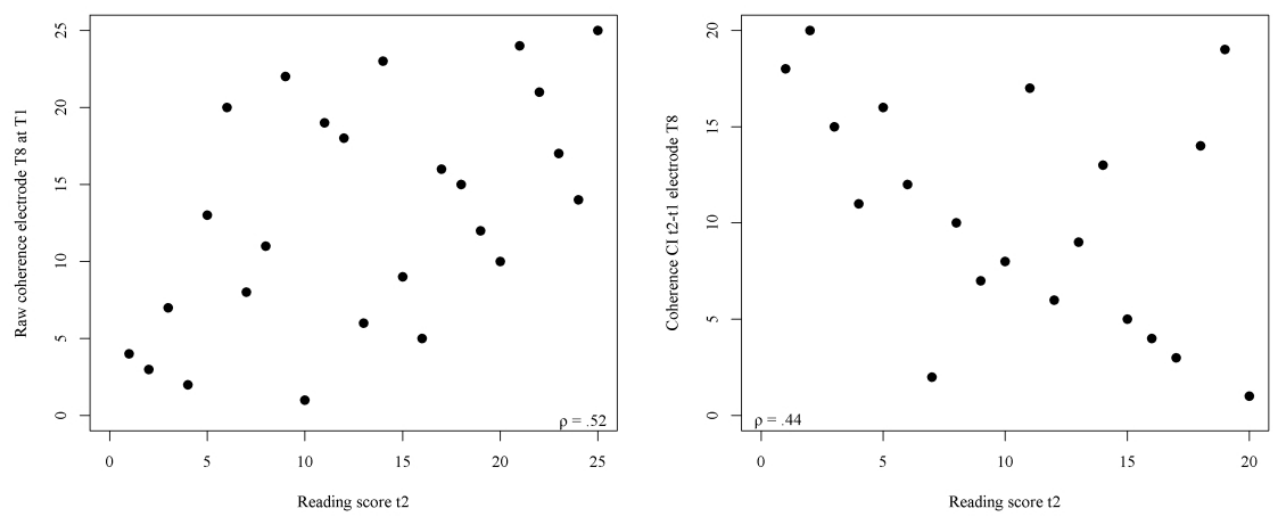

Figure 3. Spearman ranked correlation between speech-brain coherence and reading achievement at $t 2$. The left panel shows the correlation with the raw coherence values and the right panel, the correlation with the Coh from t1 to t2. Note that the values are represented in ranks.

$330 \times 152 \mathrm{~mm}(300 \times 300$ DPI $)$ 


\section{Supplementary materials}

Table S1. Individual per minute efficiency scores (correct words/time * 60 ) in the reading tasks. The Item reading column is the average of the Word and Pseudoword reading subtasks. The last column shows the efficiency scores in the Reading composite measure (the average of the ..Item and Text reading tasks)

\begin{tabular}{|c|c|c|c|}
\hline Subj & $\begin{array}{l}\text { Item } \\
\text { reading }\end{array}$ & $\begin{array}{c}\text { Text } \\
\text { reading }\end{array}$ & Composite \\
\hline 1 & 13.44 & 10.63 & 12.03 \\
\hline 2 & 19.82 & 22.67 & 21.24 \\
\hline 3 & 31.93 & 44.39 & 38.16 \\
\hline 4 & 8.84 & 20.81 & 14.82 \\
\hline 5 & 12.72 & 11.7 & 12.21 \\
\hline 6 & 15.78 & 8.93 & 12.36 \\
\hline 7 & 5.16 & 5.39 & 5.27 \\
\hline 8 & 22.30 & 46.11 & 34.20 \\
\hline 9 & 13.35 & 10.75 & 12.05 \\
\hline 10 & 11.64 & 13.75 & 12.70 \\
\hline 11 & 27.59 & 32.85 & 30.22 \\
\hline 12 & 20.57 & 29.23 & 24.90 \\
\hline 13 & 5.74 & 7.69 & 6.72 \\
\hline 14 & 20.38 & 28.40 & 24.39 \\
\hline 15 & 5.03 & 10.05 & 7.54 \\
\hline 16 & 17.30 & 8.96 & 13.13 \\
\hline 17 & 25.48 & 35.85 & 30.66 \\
\hline 18 & 11.03 & 10.16 & 10.60 \\
\hline 19 & 26.86 & 37.38 & 32.12 \\
\hline 20 & 6.15 & 5.93 & 6.04 \\
\hline 21 & 9.90 & 19.74 & 14.82 \\
\hline 22 & 24.68 & 24.76 & 24.72 \\
\hline 23 & 12.01 & 18.87 & 15.44 \\
\hline 24 & 15.12 & 20.81 & 17.97 \\
\hline 25 & 25.03 & 40.59 & 32.81 \\
\hline 26 & 22.86 & 17.61 & 20.24 \\
\hline 27 & 10.53 & 10.36 & 10.45 \\
\hline 28 & 8.82 & 6.64 & 7.73 \\
\hline 29 & 24.74 & 27.23 & 25.98 \\
\hline 30 & 17.09 & 21.15 & 19.12 \\
\hline 31 & 9.15 & 10.26 & 9.70 \\
\hline
\end{tabular}

Subj $=$ Subject 
Table S2. Partial Spearman correlations between reading achievement at $t_{2}$ and the different measures of brain-speech coherence at $0.5 \mathrm{~Hz}$ in parietal electrodes

a. Raw coherence

\begin{tabular}{c|c|c|c|c} 
& & & Reading $\mathbf{t}_{\mathbf{2}}$ & \\
\cline { 1 - 3 } Electrode & Testing time & $\mathbf{n}$ & $\boldsymbol{\rho}$ & $\boldsymbol{p}$ \\
\hline P7 & \multirow{2}{*}{$\mathrm{t}_{1}$} & \multirow{2}{*}{25} & .32 & .12 \\
\cline { 1 - 3 } P8 & & .16 & .45 \\
\cline { 1 - 3 } P7 & \multirow{2}{*}{$\mathrm{t}_{2}$} & \multirow{2}{*}{24} & .16 & .45 \\
\cline { 1 - 3 } P8 & & & .18 & .38
\end{tabular}

b. $\Delta$ Coh from $t_{1}$ to $t_{2}$

\begin{tabular}{cccc} 
& \multicolumn{3}{c}{ Reading $\mathbf{t}_{\mathbf{2}}$} \\
\cline { 2 - 4 } Electrode & $\mathbf{n}$ & $\boldsymbol{\rho}$ & $\boldsymbol{p}$ \\
\hline P7 & 19 & .19 & .44 \\
P8 & & .16 & .50 \\
\hline
\end{tabular}

